\title{
Autonomous 3D Object Modeling by a Humanoid Using Optimization Algorithms to Generate Whole-Body Postures Related to the Best Viewpoints
}

\author{
Torea FOISSOTTE ${ }^{1,2}$, Olivier STASSE ${ }^{2}$ and Abderrahmane KHEDDAR ${ }^{1,2}$ \\ ${ }^{1}$ CNRS-LIRMM \\ ${ }^{2}$ CNRS-AIST JRL, UMI 3218/CRT
}

\begin{abstract}
We present our original algorithm to find successive viewpoints around an unknown object in order to build its visual representation. A derivative-free optimization method, NEWUOA, and a gradient-based method, FSQP, are used to generate whole-body postures by taking into account visual features as well as constraints related to the use of a humanoid. We also incorporate a basic sampling of the configuration space to be able to cope with problems of local minima. This method has been tested on simulation with a HRP-2 humanoid robot on different objects varying in size and complexity.
\end{abstract}

Key Words: Next-Best-View, Humanoid, Autonomous, NEWUOA, FSQP

\section{Introduction}

\subsection{Context}

This work is a step toward the improvement of autonomous interaction between multi-purpose robots and their environment. We consider the ability to acquire new knowledge dynamically to be of particular interest when such robots are conducting tasks in collaboration with humans outside of controlled space. More specifically the work presented aims at building a visual representation of an unknown object which is perceived by a humanoid robot in a partially known environment, see Fig. 1.

The model created should allow a fast and efficient detection and recognition of the object when it is encountered in different conditions. It should also be designed to facilitate manipulation tasks with the objects. To address these two requirements, two representations of the object are constructed: a set of features, such as SIFT or SURF, for fast object recognition purposes and an occupancy grid, updated through stereo vision, to retrieve the 3D shape.

The perception of the object is done using stereo cameras embedded in the robot head. Thus a whole-body posture addressing related constraints needs to be generated each time a viewpoint of the object is required.

\subsection{Background}

Our view planning problem is directly related to the Next-Best-View research field which has been surveyed few times ([1], [2]). Though this field has been active for long, the particular constraints encountered when using a humanoid robot have not been addressed. Most works focus on the vision problem and/or assume a controlled sensing environment. Few works, like [3], consider the problem of the configuration space of the sensor but they are dealing with robots with far fewer degrees of freedom.

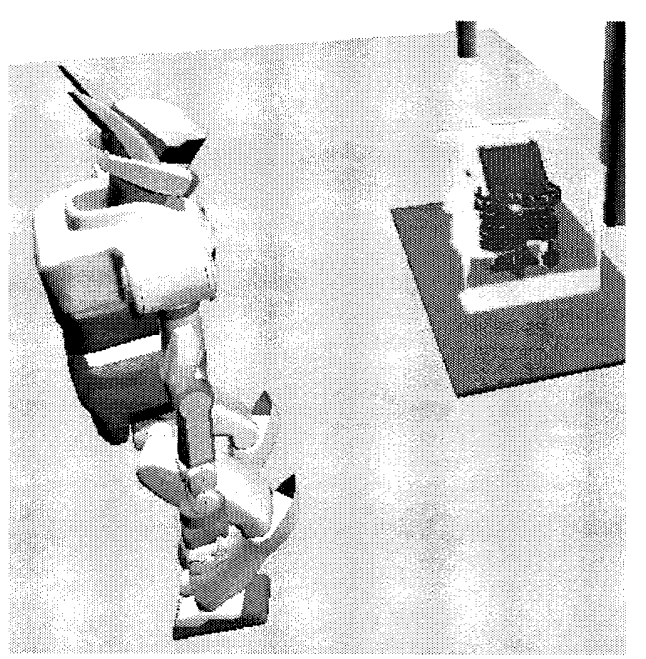

Fig. 1 HRP-2 constructing the model of a tree house

\subsection{Contribution}

Contrasting with previous methods, our approach considers the constraints on the robot such as stability, joint limits, self-collisions and collisions with the environment, in addition to the visual features of the object in order to choose successive viewpoints.

The posture generator (PG) presented in [4] uses FSQP, a gradient descent based optimization method, to generate whole-body postures addressing the constraints listed above. A previous work [5] attempt to include the estimation of visual features in the posture generator but the formulation introduced results in discontinuities in the gradient and thus FSQP cannot converge.

Our solution, introduced in [6], uses NEWUOA [7], a derivative-free optimization method, to find an optimum viewpoint pose for the camera depending on visual features and some of the constraints relative to the robot. The PG is then called with the obtained camera pose translated as a constraint on the robot head in order to generate a 
whole-body posture.

Section 2 details our algorithm while simulation results are presented in section 3 .

\subsection{Viewpoint selection}

To select successive viewpoints in order to model the entire object, it is necessary to quantify and locate the amount of occluded data that remains to be perceived. This is done similarly to [8] and [9] by using an occupancy grid and the space carving algorithm. An example of occupancy grid is shown in Fig. 1 with perceived voxels in blue and occluded voxels in green. The occupancy grid and the robot camera view frustum are simulated using an OpenGL implementation.

Our aim is then to choose a viewpoint which maximizes the amount of previously occluded data visible $N v$ while taking into account several constraints on the cameras:

- Minimum distance from the object's voxels: it is necessary to create a disparity map using the stereo rig inside the robot head.

- Height, rotation for roll and rotation for pitch in defined intervals: these intervals represent what can be obtained considering the humanoid characteristics and joints limits.

- A minimum number of previously detected features must be visible: this constraint on the camera view frustum is used to correct possible positioning errors of the robot relatively to the object by matching detected features.

- The camera pose is different than all poses in a list of forbidden poses: in the cases where it is not possible to generate a posture with the required viewpoint, a new one must be found. To ensure the algorithm does not fall back on the same viewpoint, we create a list of those to avoid.

These constraints are formulated as continuous functions and are summed together with $-N v$ to obtain an objective function to minimize. Such function is not derivable and thus we use NEWUOA, an optimization method that can find a minimum through a deterministic adaptive sampling of the function. This method is adapted to simple objective functions or local searches but in our case, it is possible to get stuck in local minima. To overcome this problem, we make a rough uniform sampling of the configuration space, launch the NEWUOA search at each sampled position and keep the best viewpoint found.

\subsection{Posture generation}

When a viewpoint is selected, the Posture Generator is launched in order to find a whole-body posture addressing all the following constraints:

- The humanoid head must be set to the position and orientation deduced from the desired viewpoint for the cameras.

- The posture obtained is statically stable: this is a requirement to grab better images with the cameras.

- The feet are flat on the floor: this improves the stability especially in real experiments compared to simulation.

- Joints limits are respected.

- There are no self-collisions.

- There are no collisions with the environment: this necessitates some knowledge about the possible obstacles around the object to model.

If the PG cannot converge, the required viewpoint is entered into the forbidden poses list and a new viewpoint is searched for. When the PG converges, the humanoid is moved to the result pose and the object is perceived by the stereo cameras. Visual features are detected on the object, matched with previously found features, and saved in the set corresponding to the object.

The model is considered complete if the number of occluded voxels in the model gets below a defined level or remains stable for a defined number of iterations.

\section{Simulation Results}

Our algorithm has been tested in simulation for the autonomous reconstruction of 14 objects with different shape complexity and sizes varying between 0.2 to 6 meters. Fig. 2 illustrates the modeling process of a 6 meters high open house which finished after 8 iterations.

On an average computer and with an occupancy grid of size $64 \times 64 \times 64$, it takes typically less than 5 seconds for NEWUOA to find a viewpoint. Thus depending on the number of sampled starting positions, it takes few seconds to few minutes to select a better viewpoint. The PG computation time is in the order of milliseconds and rarely up to few seconds when some problems of convergence are encountered.

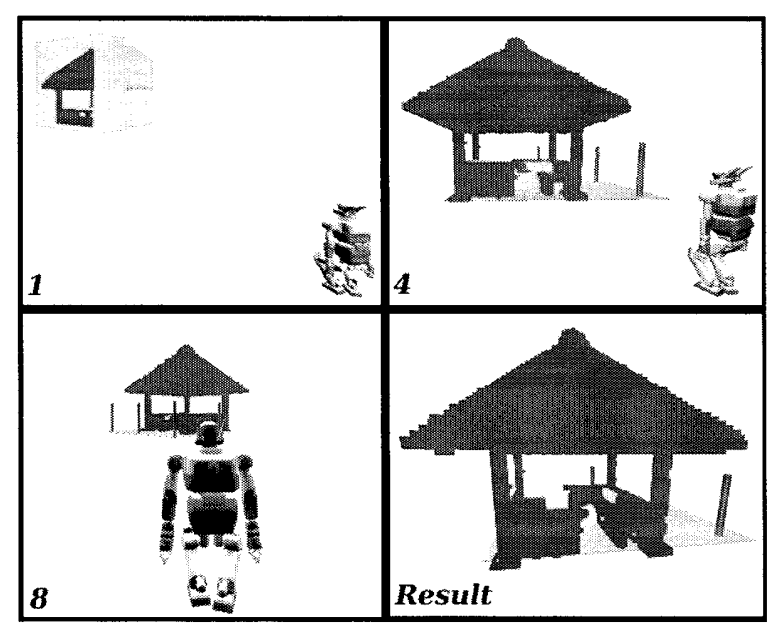

Fig.2 Modeling iterations of an open house 
Further details about the algorithm formulation and results have been presented in [6] and [10].

\section{Conclusion}

An original method to address the Next-Best-View problem by taking into account conditions and constraints related to the use of a humanoid robot has been presented. An autonomous and efficient acquisition of a model for an unknown object is possible by using a two-steps approach: first use the visual features and the camera constraints to find a desired viewpoint, and then generate a whole-body posture using the constraints related to the humanoid.

The performance has been demonstrated through simulation for a great variety of objects.

Currently we are integrating different works in pattern generation and path planning in order to test this approach in real experiments.

\section{Acknowledgement}

This work is partially supported by grants from the ROBOT@CWE EU CEC project, Contract No.34002 under the 6th Research program [www.robot-at-cwe.eu].

The visualization of the experimental setup relied on the AMELIF framework presented in [11].

\section{References}

[1] K. Tarabanis, P. Allen, and R. Tsai, "A survey of sensor planning in computer vision," in IEEE Transactions on Robotics and Automation, vol. 11, pp. 86-104, 1995.

[2] W. Scott, G. Roth, and J. Rivest, "View planning for automated three dimensional object reconstruction and inspection," ACM Computing Survey, vol. 35-1, pp. 64-96, 2003.

[3] Y. Huang and K. Gupta, "An Adaptive Configuration-space and Work-space Based Criterion for View Planning," in IEEE/RSJ International Conference on Intelligent Robots and Systems (IROS), 2-6 August, pp. 3366-3371, 2005

[4] A. Escande, A. Kheddar, and S. Miossec, "Planning support contact points for humanoid robots and experiments on hrp-2," in IEEE/RSJ International Conference on Intelligent Robots and Systems (IROS), pp. $2974-2979,2006$.

[5] T. Foissotte, O. Stasse, A. Escande, and A. Kheddar, "A next-best-view algorithm for autonomous $3 \mathrm{~d}$ object modeling by a humanoid robot," in IEEE RAS/RSJ Conference on Humanoids Robots, 1-3 Dec., 2008.

[6] T. Foissotte, O. Stasse, A. Escande, P.-B. Wieber, and A. Kheddar, "A two-steps next-best-view algorithm for autonomous $3 \mathrm{~d}$ object modeling by a humanoid robot," in IEEE ICRA Proceedings, 2009.

[7] M. Powell, "The newuoa software for unconstrained optimization without derivatives," University of Cambridge, Tech. Rep. DAMTP Report 2004/NA05, 2004.

[8] C. Connolly, "The determination of next best views," in IEEE International Conference on Robotics and Automation, vol. 2, pp. 432-435, 1985.

[9] J. Banta, Y. Zhien, X. Wang, G. Zhang, M. Smith, and M. Abidi, "A best-next-view algorithm for three-dimensional scene reconstruction using range images," in Proceedings SPIE, pp. 418-429, 1995.

[10] T. Foissotte, O. Stasse, P.-B. Wieber, and A. Kheddar, "Using NEWUOA to drive the autonomous visual modeling of an object by a Humanoid Robot", submitted to IEEE ICIA, 2009. Unpublished.

[11] P. Evrard, F. Keith, J.-R. Chardonnet, and A. Kheddar, "Framework for haptic interaction with virtual avatars," in 17 th IEEE International Symposium on Robot and Human Interactive Communication (IEEE RO-MAN 2008), 2008 